\title{
EFICÁCIA DO BYPASS GÁSTRICO NO COMBATE A DOENÇAS METABÓLICAS: UMA REVISÃO SISTEMÁTICA DA LITERATURA
}

\author{
José António Barros Monteiro \\ Unidade de Cuidados Continuados em Cinfães \\ Maria Helena Pimentel \\ Unidade de Investigação em Ciências da Saúde: \\ Enfermagem (UICISA:E), Instituto Politécnico de Braganca, Portugal.
}

Recepción Artículo: 16 marzo 2021

Admisión Evaluación: 16 marzo 2021

Informe Evaluador 1: 20 marzo 2021

Informe Evaluador 2: 24 marzo 2021

Aprobación Publicación: 26 marzo 2021

\section{RESUMO}

Atualmente, a obesidade é um dos principais problemas de saúde pública, sendo considerada uma doença crónica e ao mesmo tempo um fator de risco para o desenvolvimento de outras doenças crónicas, associa-se a um conjunto de alterações metabólicas, nomeadamente a hipertensão arterial e a diabetes mellitus tipo 2. 0 Bypass gástrico é uma cirurgia muito utilizada por conseguir atingir perdas de $40 \%$ do peso inicial, sendo realizada por vídeo-laparoscopia ou por via convencional.

Objetivo: Identificar nos diversos estudos científicos evidências que comprovem que no âmbito da cirurgia bariátrica, o bypass gástrico é um método cirúrgico eficaz no combate a doenças metabólicas, nomeadamente a hipertensão arterial e a diabetes mellitus tipo 2. Métodos: Trata-se de um estudo, delineado conforme os procedimentos da revisão sistemática da literatura, orientado pelo protocolo PICO e pela seguinte questão de investigação: "Será o bypass gástrico eficaz no combate a doenças metabólicas, nomeadamente a hipertensão e a diabetes mellitus tipo 2?" Resultados: Relativamente à diabetes mellitus tipo 2 verificou-se uma prevalência que variou de $25 \%$ a $86,8 \%$. Já os dados referentes à hipertensão arterial a HTA variou de $12 \%$ a $99,5 \%$. Todos os artigos analisados concluíram que o bypass gástrico é um bom método cirúrgico para perda de peso e melhoria da qualidade de vida. Conclusão: Nos casos em que as alterações comportamentais e terapia farmacológica se mostraram ineficazes, a cirurgia bariátrica demonstrou ser uma peçachave no tratamento da diabetes mellitus tipo 2 e hipertensão arterial em indivíduos obesos, podendo mesmo causar a sua remissão completa. 0 bypass gástrico mostrou ser um bom método cirúrgico, apresentando resultados a nível de benefício/risco, tendo um elevado grau de eficácia e baixas 
complicações. Embora eficaz implica alguns riscos sendo necessário um estudo personalizado para cada doente de modo a calcular o benefício/risco individualmente.

Palavras chave: obesidade; hipertensão arterial; diabetes mellitus; cirurgia bariátrica; bypass gástrico.

\section{ABSTRACT}

Efficacy of Gastric Bypass in the fight against metabolic diseases: A Systematic Literature Review. Currently, obesity is one of the main public health problems, being considered a chronic disease and at the same time a risk factor for the development of other chronic diseases, it is associated with a set of metabolic alterations, namely arterial hypertension and type 2 diabetes mellitus. Gastric bypass is a widely used surgery because it achieves losses of $40 \%$ of the initial weight, being performed by video-laparoscopy or by conventional route. Aim: Identify in the various scientific studies evidences that prove that in the scope of bariatric surgery, gastric bypass is an effective surgical method to fight metabolic diseases, namely arterial hypertension and type 2 diabetes mellitus. Methods: This is study, designed according to the procedures of the systematic literature review, and guided by the PICO protocol and the following research question: "Is gastric bypass effective in combating metabolic diseases, namely hypertension and type 2 diabetes mellitus?" Results: Regarding type 2 diabetes mellitus there was a prevalence that varied from $25 \%$ to $86.8 \%$. The data referring to arterial hypertension, in my study, the HTA varied from $12 \%$ to $99.5 \%$. All analyzed articles concluded that gastric bypass is a good surgical method for weight loss and improving quality of life. Conclusion: In cases where behavioral changes and pharmacological therapy are ineffective, bariatric surgery has proved to be a key part in the treatment of type 2 diabetes mellitus and arterial hypertension in obese individuals and may even cause complete remission. Gastric bypass proved to be a good surgical method, presenting results in terms of benefit / risk, with a high degree of effectiveness and low complications. Although it is effective it entails some risks requiring a personalized study for each patient in order to calculate the benefit / risk individually.

Keywords: obesity; artrial hypertension; diabetes mellitus; bariatric surgery; gastric bypass

\section{INTRODUÇÃO}

A obesidade é uma doença crónica, multifatorial, caracterizada por uma acumulação excessiva de gordura, resultante de um desequilíbrio entre a ingestão e 0 gasto energético, que prejudica a saúde. Atualmente, mais de metade da população adulta portuguesa tem excesso de peso, com maior proporção nos grupos mais desfavorecidos da população, sendo que para os idosos este valor ultrapassa os $80 \%$.

0 excesso de peso, que inclui a pré-obesidade e a obesidade é provavelmente o principal problema de saúde pública em Portugal, afetando mais de $50 \%$ da população adulta com implicações sérias no aparecimento e curso de diferentes patologias como a diabetes, a doença cérebro e cardiovascular, a patologia osteoarticular e a generalidade dos cancros. Doenças que no seu todo representam a principal despesa em saúde do estado português e o principal encargo do Serviço Nacional de Saúde .

A obesidade associa-se a um conjunto de alterações metabólicas descritas como a "síndrome metabólica", a qual é definida como um conjunto de anormalidades metabólicas que incluem obesidade abdominal, insulinorresistência, dislipidemia e hipertensão arterial, tornando-se num dos maiores desafios de saúde pública em todo o mundo, constituindo um fator de risco para a doença cardiovascular .

Estima-se que em 2030 , caso as tendências recentes se mantenham, $60 \%$ da população mundial padecerá de excesso de peso, 3.3 mil milhões de pessoas de sobrepeso e 1.1 mil milhões de obesidade . 
A cirurgia bariátrica é uma parte integral e devidamente estabelecida na gestão da morbilidade nos pacientes obesos. 0 crescente aumento deste tipo de intervenções realizadas em todo o mundo, intensificou e chamou a atenção para os seus efeitos a longo prazo. Os pacientes que procuram este tipo de cirurgias, por norma apresentam um extenso histórico de tentativas de perdas de peso .

A elevada prevalência do excesso de peso em Portugal requer uma intervenção mais concertada, desde a prevenção ao tratamento. 0 bypass gástrico é a operação mais praticada ("Gold standard") em cirurgia bariátrica porque é efetiva quanto à perda de peso e à redução das doenças associadas, permitindo longevidade acrescida e melhor qualidade de vida aos doentes operados. É uma técnica cirúrgica que deve ser usada em contexto de equipa especializada e multidisciplinar. Tem um efeito de controlo altamente benéfico sobre as comorbilidades, com particular destaque para a diabetes do adulto (Musella, 2016).

As cirurgias bariátricas podem ser restritivas, mal absortivas e mistas, cada uma com diferentes riscos, resultados e efeitos colaterais (Sousa, 2013). Vários estudos demonstram que os resultados do bypass são duradouros. No bypass gástrico, a ingestão alimentar não é tão limitada como nas técnicas restritivas e os estudos de qualidade de vida indicam melhor resultado do que com as técnicas restritivas puras (Musella, 2016).

Quanto à resolução das comorbilidades o efeito mais importante, sem dúvida, é sobre a Diabetes Mellitus tipo 2. Pories, citado por Musella (2016) com uma "coorte" de 608 doentes com 16 anos de follow-up, entre 1980 a 1996, obteve valores normais de glicemia em 83\% dos diabéticos e $99 \%$ de diminuição da tolerância à glicose. Este efeito, profundo e imediato, sobre o metabolismo dos hidratos de carbono desencadeou uma onda de investigação em curso sobre a possibilidade de resolver a diabetes, mesmo no doente não obeso, por via cirúrgica. Na hipertensão, o resultado é também muito significativo, mas parece, ao contrário da diabetes, correlacionar-se melhor com a perda de peso ao longo do primeiro ano. Em muitos casos não se consegue a "cura" mas, quase sempre, uma redução da medicação (Musella, 2016).

\section{OBJETIVO DA INVESTIGAÇÃO}

Identificar nos diversos estudos científicos, evidências que comprovem que no âmbito da cirurgia bariátrica, o bypass gástrico é um método cirúrgico eficaz no combate das doenças metabólicas, nomeadamente a hipertensão arterial e a diabetes mellitus tipo 2.

\section{METODOLOGIA}

Trata-se de uma investigação sob a forma de uma revisão sistemática da literatura (RSL), esta é considerada uma síntese rigorosa de todas as pesquisas relacionadas com a questão de investigação e simultaneamente síntese da informação/dados de múltiplos estudos (Sousa, 2012).

Em ciências da saúde este tipo de estudo pode trazer contribuições potenciais e constitui parte valiosa do processo de criação e organização do corpo de conhecimento sobre determinado tema (Donato \& Donato, 2019).

Sousa (2012) refere que a prática baseada na evidência é composta por quatro etapas que representam uma revisão sistemática. Essas quatro etapas são: identificação do problema, formulação de perguntas de investigação, pesquisa de evidência científica e avaliação da evidência disponível.

Após a seleção dos artigos a incluir na RSL utilizámos como referência a estratégia PICO que representa um acrónimo para Utente, Intervenção, Comparação e "Outcomes". Por não se aplicar excluímos a comparação passando a PIO. 
EFICÁCIA DO BYPASS GÁSTRICO NO COMBATE A DOENĢAS METABÓLICAS: UMA REVISÃO SISTEMÁTICA DA LITERATURA

\section{Tabela 1 - Resumo da estratégia PIO}

\begin{tabular}{ll}
\hline Participantes & Utentes que realizaram bypass gástrico. \\
Intervenção & $\begin{array}{l}\text { Avaliação da eficácia do bypass gástrico no combate a } \\
\text { doenças metabólicas, nomeadamente, a hipertensão arterial } \\
\text { e a diabetes mellitus tipo } 2 .\end{array}$ \\
Outcomes & $\begin{array}{l}\text { Eficácia do bypass gástrico perante doenças metabólicas, } \\
\text { nomeadamente a hipertensão arterial e a diabetes mellitus } \\
\text { tipo } 2 .\end{array}$ \\
\hline
\end{tabular}

Para a concretização da presente investigação, foram definidos procedimentos específicos de análise dos artigos, respeitando critérios de seleção, quer de inclusão quer de exclusão, como demonstra a tabela seguinte.

Tabela 2 - Critérios de seleção/inclusão e exclusão dos estudos a selecionar segundo a estratégia PIO

\begin{tabular}{|ll|}
\hline \multicolumn{1}{|c}{ Critérios de Inclusão } & \multicolumn{1}{c|}{ Critérios de Exclusão } \\
\hline Artigos publicados nos últimos 5 anos; & Artigos de revisão bibliográfica, \\
Estudos escritos em língua portuguesa, & Protocolos de estudo sem avaliação final; \\
espanhola e inglesa; & Artigos de opinião. \\
Artigos disponíveis em versão integral; & \\
Participantes com idade $\geq 18$ anos. & \\
\hline
\end{tabular}

Com recurso às plataformas PubMed e Scielo como ferramenta de trabalho foram analisados os resultados obtidos na pesquisa refinada do tema, de forma a obter um número de artigos científicos que respondessem à questão de investigação e objetivos propostos, posteriormente analisados detalhadamente. Podem ainda ser utilizados operadores booleanos representados por "AND", "OR" e "NOT" durante a pesquisa de evidências em plataformas online, utilizando mais do que uma palavra. Estes operadores facilitam a procura de evidências.

0 operador "AND" é usado quando o investigador procura evidências que utilizem as palavras em conjunto, neste caso, pretendia-se pesquisar sobre bypass gástrico, hipertensão arterial e diabetes mellitus tipo 2, com "gastric bypass AND arterial hypertension AND diabetes mellitus type 2". No fundo este operador estabelece uma combinação restrita de palavras-chave para a pesquisa.

0 operador "OR" representa uma combinação aditiva combinando, neste estudo, os conceitos "gastric bypass", "arterial hypertension" e "diabetes mellitus type 2" de forma a obter evidências que contivessem pelo menos uma das palavras-chave selecionadas, não necessitando da presença dos três termos nas evidências resultantes da pesquisa. 0 operador "NOT" é utilizado para excluir termos não desejados. A figura 1 representa o fluxograma da investigação. 


\section{Figura 1 - Fluxograma de Investigação}

\begin{tabular}{|c|c|c|c|c|c|}
\hline $\begin{array}{l}\text { A } \\
\text { rt } \\
\text { ig } \\
\text { o }\end{array}$ & Autor e Ano & $\begin{array}{l}\text { Tipo de } \\
\text { Estudo }\end{array}$ & Objetivo do Estudo & Amostra & País \\
\hline 1 & $\begin{array}{l}\text { (Rolim, Cruz, Campos, } \\
\text { \& Ferraz, 2018) }\end{array}$ & $\begin{array}{l}\text { Quantitativo, } \\
\text { experimental }\end{array}$ & $\begin{array}{l}\text { Avaliar a evolução ponderal, nutricional } \\
\text { e a qualidade de vida da amostra, após } \\
\text { dez anos de Bypass Gástrico. }\end{array}$ & $\begin{array}{l}42 \text { utentes } \\
\text { classificados com } \\
\text { dificuldades } \\
\text { socioeconómicas. }\end{array}$ & Brasil \\
\hline 2 & $\begin{array}{l}\text { (Ruiz-Mar, Ruelas- } \\
\text { Ayala, Ornelas-Oñate, } \\
\text { \& Ramirez-Velasquez, } \\
\text { 2019) }\end{array}$ & $\begin{array}{l}\text { Quantitativo, } \\
\text { experimental }\end{array}$ & $\begin{array}{l}\text { Descrever a técnica e os resultados, após } \\
\text { um ano, do Bypass gástrico com uma } \\
\text { anastomose e os seus efeitos na perda de } \\
\text { peso e na remissão de comorbidades. }\end{array}$ & $\begin{array}{l}51 \text { utentes } \\
\text { mulheres e } 83 \\
\text { homens) com idade } \\
\text { média } 43,8 \pm 9,3 \\
\text { anos, peso médio } \\
125 \pm 31 \mathrm{~kg} \text { e IMC } \\
\text { médio } 55,8 \pm 12 \\
\mathrm{~kg} / \mathrm{m}^{2}\end{array}$ & México \\
\hline 3 & (Schnor, et al., 2016) & $\begin{array}{l}\text { Quantitativo, } \\
\text { experimental }\end{array}$ & $\begin{array}{l}\text { Investigar a associação entre } \\
\text { polimorfismos genéticos relacionados à } \\
\text { obesidade na prevalência de HTA e } \\
\text { DMt2 no pré-operatório e } 1 \text { ano após a } \\
\text { cirurgia de bypass gástrico. }\end{array}$ & $\begin{array}{l}351 \text { utentes do sexo } \\
\text { feminino, entre os } \\
20 \text { e os } 50 \text { anos de } \\
\text { idade. }\end{array}$ & Brasil \\
\hline 4 & (Ke, et al., 2017) & $\begin{array}{l}\text { Quantitativo, } \\
\text { experimental }\end{array}$ & 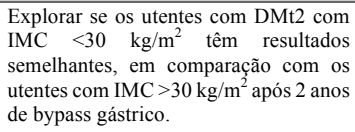 & $\begin{array}{l}70 \text { utentes } \quad \text { com } \\
\text { DMt2 não } \\
\text { controlados: } \\
47 \mathrm{IMC}<30 \mathrm{~kg} / \mathrm{m}^{2} \text {; } \\
23 \mathrm{IMC}>30 \mathrm{~kg} / \mathrm{m}^{2}\end{array}$ & China \\
\hline 5 & $\begin{array}{l}\text { (Ikramuddin, et al., } \\
\text { 2016) }\end{array}$ & $\begin{array}{l}\text { Quantitativo, } \\
\text { experimental }\end{array}$ & $\begin{array}{l}\text { Avaliar os resultados dos riscos de } \\
\text { controle e tratamento da diabetes } 2 \text { anos } \\
\text { após a adição do bypass gástrico ao } \\
\text { estilo de vida intensivo e ao } \\
\text { aconselhamento médico. }\end{array}$ & 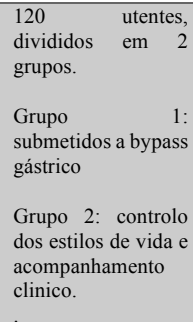 & $\begin{array}{l}\text { EUA } \\
\text { Taiwan }\end{array}$ \\
\hline 6 & (Salminen, et al., 2018) & $\begin{array}{l}\text { Quantitativo, } \\
\text { experimental }\end{array}$ & $\begin{array}{l}\text { Determinar se a cirurgia gastrectomia } \\
\text { laparoscópica e o bypass gástrico em Y } \\
\text { de Roux são equivalentes à perda de } \\
\text { peso, após } 5 \text { anos em utentes com } \\
\text { obesidade mórbida. }\end{array}$ & $\begin{array}{l}240 \text { utentes }(69,6 \% \\
\text { mulheres e } 30,4 \% \\
\text { homens), com idade } \\
\text { média de } 48 \text { anos e } \\
\text { IMC médio de } 45,9 . \\
\text { Separados em dois } \\
\text { grupos, } 121 \text { no } \\
\text { grupo gastrectomia } \\
\text { vertical e } 119 \text { no } \\
\text { grupo bypass } \\
\text { gástrico. }\end{array}$ & Finlândia \\
\hline
\end{tabular}


EFICÁCIA DO BYPASS GÁSTRICO NO COMBATE A DOENĢAS METABÓLICAS:

UMA REVISÃO SISTEMÁTICA DA LITERATURA

\section{RESULTADOS}

A Tabela 3 e 4 apresentam de forma sintetizada os 6 trabalhos científicos incluídos no presente estudo.

Tabela 3 - Artigos científicos incluídos no estudo

\section{Artigos Cientificos}

Palavras-chave: Bypass Gástrico; Obesidade; Hipertensão Arterial; Diabetes

Mellitus tino 2

Scielo (13)

Pubmed (221)

Artigos Cientificos Publicados nos últimos 5 anos (2015-2020)

Scielo (5)

Pubmed (110)

Artigos Cientificos nos Idiomas Português, Inglês e Espanhol e em Texto Integral

Scielo (5)

Pubmed (30)

Excluídos pela Leitura do Título e Resumo

Scielo (5)

Pubmed (5)

Incluídos pela Leitura e Análise Integral dos Artigos

Scielo (3)

Pubmed (3)

Tabela 4 - Resumo dos principais resultados e conclusões dos artigos analisados.

\begin{tabular}{|c|c|c|}
\hline Artigo & Resultados & Conclusões \\
\hline 1 & $\begin{array}{l}\text { Inicialmente, } 59,5 \% \text { da amostra } \\
\text { apresentava HTA e } 9,5 \% \text { DMt } 2 \text {. } \\
\text { Relativamente à HTA, após } 10 \text { anos de } \\
\text { estudo, observou-se uma remissão de } \\
66 \% \text {. Aos } 6 \text { meses pós-operatório a } \\
\text { taxa de remissão foi semelhante à } \\
\text { observada após } 10 \text { anos. } \\
\text { Na DMt2 foi observada uma taxa de } \\
\text { remissão de } 50 \% \text {, o baixo rendimento } \\
\text { económico dificulta } \\
\text { acompanhamento pós cirúrgico em } \\
55,6 \% \text {. }\end{array}$ & $\begin{array}{l}\text { O estudo demonstrou que o } \\
\text { bypass gástrico é eficaz na } \\
\text { perda de peso (mais de } 50 \% \text { ), } \\
\text { porém a falta de } \\
\text { acompanhamento a longo } \\
\text { prazo comprometeu o resultado } \\
\text { final. } \\
\text { Perante as diversas limitações } \\
\text { socioeconómicas, o bypass } \\
\text { gástrico apresenta níveis } \\
\text { satisfatórios, melhorando a } \\
\text { qualidade de vida da amostra. }\end{array}$ \\
\hline
\end{tabular}




\begin{tabular}{|c|c|c|}
\hline 2 & $\begin{array}{l}\text { Inicialmente, } 43,1 \% \text { da amostra } \\
\text { apresentava HTA e } 64,7 \% \text { DMt } 2 \text {. } \\
\text { Após um ano, relativamente à HTA, } \\
\text { observou-se uma remissão de } 37 \% \text {. Na } \\
\text { DMt2 uma taxa de remissão de } 57 \% \text {. }\end{array}$ & $\begin{array}{l}\text { Tendo este estudo um } \\
\text { acompanhamento dos utentes } \\
\text { durante um ano após a cirurgia, } \\
\text { os resultados neste curto prazo } \\
\text { parecem promissores. }\end{array}$ \\
\hline 3 & $\begin{array}{l}\text { Inicialmente, } 57 \% \text { da amostra } \\
\text { apresentava HTA e } 22 \% \text { DMt } 2 . \\
\text { Após um ano, relativamente à HTA, } \\
\text { observou-se uma remissão de } 99,5 \% \text {. Já } \\
\text { na DMt2 uma taxa de remissão de } \\
86,8 \% \text {. }\end{array}$ & $\begin{array}{l}\text { Este estudo não avaliou a perda } \\
\text { de peso, o que dificulta em } \\
\text { parte a compreensão dos } \\
\text { valores de remissão. Após um } \\
\text { ano de investigação, em } \\
\text { comparação com os outros } \\
\text { estudos, os resultados } \\
\text { apresentados em relação à } \\
\text { HTA e à DMt2 são muito } \\
\text { satisfatórios. }\end{array}$ \\
\hline 4 & $\begin{array}{l}70 \text { utentes com DMt2 não controlada } \\
\text { foram divididos em dois grupos: } 47 \\
\text { com IMC }<30 \mathrm{~kg} / \mathrm{m}^{2} \text { e } 23 \text { com IMC } \\
>30 \mathrm{~kg} / \mathrm{m}^{2} \text {. } \\
\text { Após dois anos, a remissão completa da } \\
\text { DMt } 2 \text { em } 28,2 \% \text { no grupo IMC }<30 \\
\mathrm{~kg} / \mathrm{m}^{2} \text {, significativamente menor do } \\
\text { que no grupo IMC }>30 \mathrm{~kg} / \mathrm{m}^{2} \text {, no qual }\end{array}$ & $\begin{array}{l}\text { Este estudo confirmou que o } \\
\text { bypass gástrico é seguro e que } \\
\text { pode ser útil no tratamento de } \\
\text { baixo IMC em utentes com } \\
\text { DMt2. } \\
\text { Os autores concluem que o } \\
\text { bypass gástrico pode melhorar } \\
\text { os fatores de risco } \\
\text { cardiovascular, que por sua vez }\end{array}$ \\
\hline & $\begin{array}{l}\text { a taxa de remissão foi de } 57,9 \%(\mathrm{p}= \\
0,029) \text {. } \\
\text { Não se verificou uma diferença } \\
\text { significativa na melhoria (HbA1c } \\
<7,0 \%) \text { da DMt } 2 \text { entre grupos. O que } \\
\text { significa que os utentes no grupo IMC } \\
<30 \mathrm{~kg} / \mathrm{m}^{2} \text { também beneficiam da } \\
\text { cirurgia de bypass gástrico, mesmo } \\
\text { com menor remissão completa do } \\
\text { diabetes. }\end{array}$ & $\begin{array}{l}\text { podem melhorar a qualidade de } \\
\text { vida dos utentes. }\end{array}$ \\
\hline
\end{tabular}




\begin{tabular}{|c|c|c|}
\hline 5 & $\begin{array}{l}\text { Todos os utentes apresentavam DMt2 } \\
\text { pelo menos durante } 6 \text { meses antes do } \\
\text { início do estudo. } 53 \% \text { dos participantes } \\
\text { eram hipertensos, embora } 71 \% \\
\text { usassem medicação anti-hipertensiva. } \\
\text { Após dois anos de estudo, } 25 \% \text { dos } \\
\text { utentes do grupo submetido a bypass } \\
\text { gástrico apresentaram remissão total da } \\
\text { diabetes e } 42 \% \text { tiveram remissão } \\
\text { parcial, enquanto nenhum utente do } \\
\text { grupo do controlo dos estilos de vida e } \\
\text { acompanhamento clinico obteve } \\
\text { remissão. } \\
\text { Os utentes no grupo do bypass gástrico } \\
\text { apresentaram tensão arterial média } \\
\text { mais baixa do que os utentes do grupo } \\
\text { do controlo dos estilos de vida e } \\
\text { acompanhamento clinico aos } 24 \text { meses, } \\
\text { mas sem diferença entre os grupos na } \\
\text { hipertensão arterial sistólica (menor } \\
\text { que } 130 \text { mmHg). Apenas } 12 \% \text { dos } \\
\text { submetidos a bypass gástrico atingiram } \\
\text { o objetivo de reduzir ou cessar a } \\
\text { medicação. A perda de peso foi } \\
\text { substancialmente maior neste grupo. }\end{array}$ & $\begin{array}{l}\text { Este estudo confirmou que o } \\
\text { grupo do bypass gástrico } \\
\text { diminuiu a medicação para } \\
\text { atingir os objetivos propostos, } \\
\text { ressaltando o aparente } \\
\text { benefício metabólico da } \\
\text { cirurgia. } \\
\text { A melhoria no controle } \\
\text { metabólico no grupo do bypass } \\
\text { gástrico foi atribuída ao melhor } \\
\text { controlo glicémico, do qual } \\
75 \% \text { atingiu a meta de HbA1c } \\
\text { menor que } 7,0 \% \text {, enquanto só } \\
24 \% \text { do segundo grupo obteve } \\
\text { o mesmo resultado. } \\
\text { As possíveis explicações para } \\
\text { uma menor taxa de remissão da } \\
\text { diabetes, incluem maior } \\
\text { gravidade da doença (HbA1c } \\
\text { média } 9,6 \%) \text { e maior duração } \\
\text { da diabetes (8 a } 9 \text { anos). } \\
\text { As evidências sugerem que a } \\
\text { cirurgia melhora levemente a } \\
\text { hipertensão arterial mas sem } \\
\text { benefício para o colesterol. } \\
\text { As anormalidades bioquímicas } \\
\text { e deficiências nutricionais } \\
\text { foram comuns no grupo do } \\
\text { bypass gástrico, apesar dos } \\
\text { requisitos de protocolo para } \\
\text { suplementação. }\end{array}$ \\
\hline 6 & $\begin{array}{l}\text { Inicialmente, } 42 \% \text { tinham DMt2 }(52 \\
\text { gastrectomia vertical e } 49 \text { bypass } \\
\text { gástrico). A remissão completa foi } \\
\text { observada em } 12 \% \text { no grupo } \\
\text { gastrectomia vertical e em } 25 \% \text { no } \\
\text { grupo bypass gástrico. Não houve } \\
\text { diferença estatística entre os grupos no } \\
\text { nível médio de glicose em jejum: } 135,1\end{array}$ & $\begin{array}{l}\text { Ambos os procedimentos } \\
\text { resultaram em perda de peso } \\
\text { sustentada, com uma perda } \\
\text { média de excesso de peso de } \\
49 \% \text { no grupo gastrectomia } \\
\text { vertical e } 57 \% \text { no grupo bypass } \\
\text { gástrico. }\end{array}$ \\
\hline
\end{tabular}


$\mathrm{mg} / \mathrm{dL}$ no grupo gastrectomia vertical e $120,7 \mathrm{mg} / \mathrm{dL}$ no grupo bypass gástrico. $\mathrm{O}$ valor médio estimado de $\mathrm{HbA1c}$ foi igual nos dois grupos $(6,6 \%)$.

A HTA, inicialmente, $71 \%$ usavam anti-hipertensivos. Após os 5 anos, $29 \%$ no grupo gastrectomia vertical e $51 \%$ no grupo bypass gástrico descontinuaram a medicação. 35\% no grupo gastrectomia vertical e $30 \%$ no grupo bypass gástrico necessitaram de menos medicação em comparação com os valores basais;
Não houve diferenças estatisticamente significantes entre os dois grupos para os desfechos secundários da remissão da DMt2, resolução da dislipidemia, melhora da QV e morbilidade tardia.

Comparado à gastrectomia vertical, o bypass gástrico resultou em melhor resolução da HTA, com base no uso de medicamentos antihipertensivos.

\section{DISCUSSÃO}

A pergunta de partida consistia em saber se o bypass gástrico é eficaz no combate de doenças metabólicas, nomeadamente, controlo da hipertensão e da diabetes mellitus tipo 2.

Nos seis artigos analisados constata-se que a HTA e a DMt2 estão muito associadas à obesidade mórbida, sendo muito prevalentes nas amostras estudadas.

Os seis artigos analisados incluíram a DMt2 enquanto que só cinco incluíram a HTA. Todos seguiram critérios de inclusão semelhantes: IMC >25, utentes obesos; idades entre os 18 e os 60 anos; diagnóstico da DMt2 ou de outras comorbilidades; bypass gástrico como método cirúrgico.

Relativamente à diabetes mellitus tipo 2 a prevalecia variava, na amostra em estudo, entre $25 \%$ a $86,8 \%$. Após cirurgia a remissão da doença, no primeiro ano, entre $37 \%$ e $99,5 \%$; nos dois anos seguintes entre $37 \%$ e $57,9 \%$; em cinco ou mais anos entre $25 \%$ e $50 \%$. A remissão tende a diminuir com o passar dos anos, o que pode significar que alguns dos utentes submetidos voltaram aos hábitos anteriores à cirurgia com tendência para aumentar de peso. A hipertensão arterial variava entre $12 \%$ a 99,5\%. Após cirurgia a tensão arterial baixou, no primeiro ano, entre $57 \%$ e $86,8 \%$; nos dois anos seguintes $12 \%$; em cinco ou mais anos entre $51 \%$ e $66 \%$. 0 estudo de chegou a idênticos resultados.

0 primeiro artigo (estudo longitudinal de 10 anos) analisou uma amostra com dificuldades socioeconómicas, $56 \%$ dos doentes não teve acompanhamento adequado após bypass, o que demonstra a falta de resposta do sistema de saúde brasileiro, situação que difere da resposta do SNS português gratuito e acessível a todos os cidadãos (DGS, 2012; 2017).

0 segundo artigo avaliou a técnica e os resultados referentes à perda de peso e à remissão de comorbilidades. Os autores referiram que o bypass gástrico é um procedimento seguro e que os resultados a curto prazo parecem promissores, no entanto, um acompanhamento a longo prazo é necessário para avaliar diferentes complicações e possíveis efeitos nutricionais . A gastrectomia vertical calibrada, no estudo de Silva et al (2017), tinha verificado bons resultados terapêuticos em termos de perda de peso, melhoria da patologia associada e da qualidade de vida.

0 terceiro artigo selecionou 12 polimorfismos genéticos relacionados com a obesidade e investigou se os mesmos estavam relacionados com a prevalência de HTA e DMt2 no pré-operatório e 1 ano após a cirurgia. 0 estudo indicou que dois polimorfismos genéticos se encontravam relacionados com a prevalência de DMt2. 0 estudo não avaliou a perda de peso o que dificultou em parte a compreensão dos valores da remissão. 
0 quarto artigo comparou duas amostras em que uma delas era composto por utentes com IMC $<30 \mathrm{~kg} / \mathrm{m}^{2}$ e outra por utentes com IMC $>30 \mathrm{~kg} / \mathrm{m}^{2}$. Este estudo confirmou que o bypass gástrico é seguro e que pode ser útil no tratamento de baixo IMC em utentes com DMt2. Musella (2016) e Sousa (2013) remetem para idêntica tendência.

0 quinto artigo dividiu a amostra em 2 grupos, um com acompanhamento dos estilos de vida, o segundo grupo além do acompanhamento dos estilos de vida o bypass gástrico. Confirmou-se que 0 grupo do bypass gástrico passou a precisar de menos medicação para controlar as doenças, ressaltando 0 aparente benefício metabólico decorrente da cirurgia. As possíveis explicações para uma menor taxa de remissão incluem maior gravidade da doença e maior duração da diabetes. Resultados concordantes com Eickhoff (2016).

0 sexto artigo, comparou o bypass com a gastrectomia vertical. Ambos os procedimentos resultaram em perda de peso, sendo os submetidos a bypass gástrico os de maior perda de peso. Não houve diferenças estatisticamente significantes entre os dois grupos para os desfechos secundários da remissão da DMt2, resolução da dislipidemia, melhoria da QV e morbilidade tardia. Comparado à gastrectomia vertical, o bypass gástrico resultou em melhor resolução da HTA. A taxa de remissão da diabetes não diferiu entre os dois procedimentos aos 5 anos, o que pode ser atribuído a possíveis diferenças na duração pré-operatória do diabetes, porque a menor duração do diabetes no início do estudo está associada a taxas de remissão a curto prazo mais favoráveis após a cirurgia bariátrica. A DGS (2012; 2017) e López (2015) reportam resultados concordantes.

Todos os artigos analisados concluíram que o bypass gástrico é um bom método cirúrgico para perda de peso e melhoria da qualidade de vida.

\section{CONCLUSÃO}

Nos seis artigos analisados, embora com métodos de avaliação e/ou intervenção diferentes, os resultados apontam no sentido de uma forte associação entre 0 bypass gástrico e a redução da HTA e da DMt2.

Nos casos em que as alterações comportamentais e terapia farmacológica se mostraram ineficazes, a cirurgia bariátrica demonstrou ser uma peça-chave no tratamento da DMt2 e HTA em indivíduos obesos, podendo mesmo causar a sua remissão completa.

0 bypass gástrico mostrou ser um bom método cirúrgico, apresentando resultados a nível de benefício/risco, tendo um elevado grau de eficácia e baixas complicações. Embora eficaz, implica alguns riscos, sendo necessário um estudo personalizado para cada doente, de modo a calcular 0 benefício/risco individualmente.

A maior parte da melhoria no controle metabólico foi atribuída a um melhor controlo glicémico. Todos os estudos realizados demonstraram resultados promissores com elevadas taxas de remissão de DMt2, mesmo em doentes com IMC $<30 \mathrm{~kg} / \mathrm{m}^{2}$.

A assiduidade por parte do utente às consultas programadas e a mudança permanente dos estilos de vida também se revelaram importantes.

Uma nova era em perspetiva, principalmente no tratamento da DMt2, embora ainda haja um longo caminho a percorrer. A comunidade científica deve estar recetiva a estas mudanças no tratamento destas doenças, até hoje, incuráveis. 0 desafio para os próximos anos será a identificação de critérios de seleção dos pacientes com indicação para tratamento cirúrgico, a melhor altura para a cirurgia e 0 aperfeiçoamento das técnicas, criando assim melhores condições de segurança.

\section{REFERENCIAS BIBLIOGRÁFICAS}

Eickhoff, Hans, Matafome, Paulo, Seiça, Raquel, \& Sousa, Francisco Castro e. (2016). Cirurgia metabólica em doentes com diabetes tipo 2. Ficção ou opção terapêutica? Revista Portuguesa de 
Cirurgia, (36), 19-28.

http://www.scielo.mec.pt/scielo.php?script=sci_arttext\&pid=S1646-69182016000100004\&Ing= pt\&tlng=pt

DGS (2012). Orientação da direção geral da saúde. Boas práticas na abordagem do doente com obesidade elegível para cirurgia bariátrica. Lisboa: Direção Geral da Saúde.

DGS (2017). Obesidade - Otimização da Abordagem Terapeutica no Serviço Nacional de Saúde. Lisboa: Direção Geral da Saúde.

Donato, H.; Donato M. (2019). Etapas na Condução de uma Revisão Sistemática, Acta Médica Portuguesa, 32(3):227D0I:10.20344/amp.11923

Ikramuddin, S., Billington, C., Lee, W.-J., Bantle, J., Thomas, A., Connett, J., . . Korner, J. (2016). Roux-en-Y gastric bypass for diabetes (the Diabetes Surgery Study): 2-year outcomes of a 5year, randomised, controlled trial. University of Minnesota, Minneapolis, The Lancet, Vol. 3 (6), 13-422. doi:10.1016/S2213-8587(15)00089-3

Ke, Z., Li, F., Chen, J., Gao, Y., Zhou, X., Sun, F.,...Tong, W. (2017). Effects of Laparoscopic Rouxen-Y Gastric Bypass for Type 2 Diabetes Mellitus: Comparison of BMI $>30$ and $<30 \mathrm{~kg} / \mathrm{m} 2$. OBES SURG 27: 3040-3047. https://doi.org/10.1007/s11695-017-2926-9

Lolio, Cecília Amaro de (1990). Epidemiologia da hipertensão arterial. Revista de Saúde Pública, 24(5): 425-432. https://doi.org/10.1590/S0034-89101990000500012

Musella, M., Apers, J., Rheinwalt, K., Ribeiro, R., Manno, E...(2016). Efficacy of Bariatric Surgery in Type 2 Diabetes Mellitus Remission: the Role of Mini Gastric Bypass/One Anastomosis Gastric Bypass and Sleeve Gastrectomy at 1 Year of Follow-up. A European survey. OBES SURG, 26 : 933-940 DOI 10.1007/s11695-015-1865-6

Rolim, Francisco Felippe de Araújo, Cruz, Fernando Santa, Campos, Josemberg Marins, \& Ferraz, Álvaro Antônio Bandeira. (2018). Repercussões em longo prazo da derivação gástrica em Y de Roux em população de baixa renda: avaliação após dez anos de cirurgia. Revista do Colégio Brasileiro de Cirurgiões, 45(4), e1916. Epub August 27.https://doi.org/10.1590/0100-6991e20181916

Ruiz-Mar, G., Ruelas-Ayala, A., Ornelas-Oñate, L., \& Ramirez-Velasquez, J. (2019). Bypass Gástrico com Somente uma Anastomose: Resultados após um Ano de Acompanhamento. ABCD Arq Bras Cir Dig 2019;32(4):e1476 D0I: /10.1590/0102-672020190001e1476

Salminen, P., Helmiö, M., Ovaska, J., Juuti, A., Leivonen, M., Peromaa-Haavisto, P., . . \& Victorzon, M. (2018). Effect of Laparoscopic Sleeve Gastrectomy vs Laparoscopic 22

RouX-en-Y Gastric Bypass on Weight Loss at 5 Years Among Patients With Morbid Obesity. JAMA, 319(3): 241-254. doi:10.1001/jama.2017.20313

Santos, C., Pimenta, C., \& Nobre, M. (2007). A Estratégia PICO para a Construção da Pergunta de Pesquisa e Busca de Evidências. Revista Latino-Americana de Enfermagem, 15(3), 508-511. https://dx.doi.org/10.1590/S0104-11692007000300023

Schnor, N., Verlengia, R., Novais, P., Crisp, A., Leite, C., Rasera-Júnior, I., \& Oliveira, M. (2016). Association of 5-HT2C (rs3813929) and UCP3 (rs1800849) gene polymorphisms with type 2 diabetes in obese women candidates for bariatric surgery. Universidade Estadual Paulista Júlio de Mesquita Filho (Unesp). Brasil: Scielo. doi:10.1590/2359-3997000000260

Silva, Soraia, Milheiro, António, Ferreira, Luis, Rosete, Manuel, Campos, José Carlos, Almeida, João, Sérgio, Mário, Tralhão, José-Guilherme, \& Sousa, Francisco Castro e. (2017). Gastrectomia Vertical Calibrada no tratamento da obesidade mórbida: Resultados a longo prazo, comorbilidades e qualidade de vida. Revista Portuguesa de Cirurgia, (40), 11-20. http://www.scielo.mec.pt/scielo.php?script=sci_arttext\&pid=S1646- 
EFICÁCIA DO BYPASS GÁSTRICO NO COMBATE A DOENÇAS METABÓLICAS:

UMA REVISÃO SISTEMÁTICA DA LITERATURA

$69182017000100004 \& \operatorname{lng}=p t \& t \operatorname{lng}=p t$.

Sousa, A.. Cirurgia bariátrica - Aspectos clínico-cirúrgicos e cuidados nutricionais inerentes. Porto: Universida de Fernando Pessoa. https://bdigital.ufp.pt/bitstream/10284/4270/1/ Cirurgia\%20Bariátrica\%20Nutrição.pdf

Sousa, H. (2012). A pratica Baseada em evidência: Uma metodologia fundamental para alunos de enfermagem. Porto: Universidade Fernando Pessoa. https://bdigital.ufp.pt/bitstream/ 10284/2790/3/T_18151.pdf 